\title{
Speed (and/ or Acceleration) Relates Space with Time - Given Complete Information of Speed (and /or Acceleration) and Space, Time Can be Completely Specified, Does Not Matter Past or Present or Future
}

\author{
Prasenjit Debnath
}

PhD Student, NIT Agartala, India

\begin{abstract}
Speed is a very important property of matter particle and force carrying particle. Any field like the Higgs field, electromagnetic field, and gravitational field has its own fixed speed. These are basically energy fields created by some kind of force carrying particles. The Higgs field which exists everywhere in the Universe provides resistance to the matter particles and hence matter particles have mass. Mass is a property of matter particle or a particular state of matter particle that comes into existence in the presence of Higgs field. The field-particle chemistry produces the property mass in the matter particles which intern produces gravity proportional to the property mass. The Higgs field exists because of a force carrying particle called the Higgs Boson. The rate of chance of states of matter is its own evolution. Every particle has its own measure of evolution and hence its own lifetime, in other words its own time. Every particle differs from other particles in lifetime or in time itself. Every particle's lifetime is actually its own measure of time. Every lifetime is absolutely unique on its own. People measure time with respect to the Earth's rotation and revolution and compare their own time with respect to other people around to conclude good time, better time, bad time, worst time etc. These are purely psychological divisions only; time is basically a time only, not a bad time or a good time.
\end{abstract}

Keyword: Good time and bad time, Higgs Field, The rate of change of States of Matter-Evolution, Higgs Boson Particle, Mass and Gravity.

\section{Introduction}

Higgs fields are small fields that go around a loop without having any edge of them. Higgs fields do not have any beginning or any end and it does hovering around its own axis with a constant speed, the half of the speed of light (approximately) which can be termed as massless speed.

Speed of light $(C)=3,00,000 \mathrm{~km} / \mathrm{sec}=1,86,000$ miles/sec.

Speed of Higgs field $(\forall)=\frac{C}{2}=1,50,000 \mathrm{~km} / \mathrm{sec}=93$, 000 miles/sec (approximately).

Every Higgs field opposes the other Higgs field nearby to have unique identity. Higgs fields create relaxed space in the Universe which is very low energy space with least stress possible. The Higgs bosons in the Higgs field are massless as they revolve at a speed of half of the speed of light which makes them massless - the massless speed $(\forall)$. This is the reason free space is massless space. When a massive enough particle like Earth interacts with the low energy small unique identity Higgs fields which opposes each other to stay unique and make highly stable space, changes their orientation of opposition and become unidirectional to display the property of gluons from bosons, these unidirectional small Higgs field starts working together to produce gravity of a massive particle, thus gravity forms in a massive particle, which gives the weight of the massive particle and hence its mass. The Earth's weight would be different if it would have another combination of matters it is made off $[1,2]$. And hence it would have different gravity too $[3,4]$. Because almost the whole atom is void $[5,6]$ and only different electromagnetic fields among atoms create different types of matter [7, 8], it hardly matters the variation of weight for different matters on a large scale $[9,10]$, although in a small scale it looks like huge variation. Because of insignificant effect on weight due to variation of matters it is made off on a large scale, Sir Isaac Newton smartly avoided the matters of a mass it is made off while calculating the gravity $[11,12]$. He knew that the variation of different matters in a mass hardly change anything in the weight of the massive particle [13, 14]. The more the mass of a particle, the more it evens out the effect of variation of matters it is made off $[15,16]$.

\section{Boson and Gluon Are Same Force Carrying Particle but Look Different At Different Circumstances}

The Higgs bosons are force carrying particles in a small Higgs field [17, 18]. Every Higgs field opposes nearby Higgs fields to preserve unique identity. Thus, each Higgs field is keeping itself unique and highly stable in space, thus, the space is normally highly stable. Every adjacent Higgs fields have opposite revolution around their own axis with a velocity half of the speed of light - the massless velocity $(\forall)$. Thus, free space is a low energy place of high stability $[19,20]$. Problem occurs when massive particle interacts with many small low energy Higgs fields. The orientation changes like that many small Higgs fields start acting 


\section{International Journal of Science and Research (IJSR) \\ ISSN (Online): 2319-7064 \\ Index Copernicus Value (2015): 78.96 | Impact Factor (2015): 6.391}

unidirectional to set up huge field which produces gravity of the massive particle. Due to the gravitational force, the massive particle displays weight of its own. Many unidirectional bosons start displaying the property of gluons. Thus, bosons and gluons are two different states of the same force carrying particle. They only look different at different circumstances

\section{Any System has its Own Space and Time}

On psychological point of view, any systems can be broadly classified into two categories, living being and non-living being [21, 22]. For a system like ant, a meter is a long distance in space and a day is a long time to live. For a system like human being, kilometer is a long distance in space and a month is a long time to live. For a system like mosquito, 7 days is the whole life cycle [23, 24]. For a system like Earth, Sun is at a long distance in space and 1000 years is a long distance in time. For a system like Milky Way, light years are a long distance in space and billions of years is a long distance in time. Every system in the Universe has its own measure of space and time. The more massive the system, the further is the distance of concern in space and the longer is the distance in time. We cannot build massive system like Earth to concern about light years and 1000 kilometers. We must work on speed that relates space with time $[25,26]$. The higher the speed, we incorporate more space in a given time. Speed (and/ acceleration) is the only factor to change in astronomy science to change the evolution (the rate of change of state of matters). The tuning to the massless velocity can give a particle zero resistance in the Higgs field. Without external disturbances, the particle can move for infinite time at a constant speed at the massless velocity $(\forall)$. The evolution in the system will be zero at the massless velocity $(\forall)$. Hence, human can travel for indefinite time around the Universe without any evolution at the massless velocity $(\forall)$.

\section{When The Momentum Is Zero}

We know that the momentum is,

\section{Momentum $=$ mass $\times$ velocity}

At the massless velocity $(\forall)$, the mass will be zero of a particle and hence the momentum of the particle will be zero too. Without external forces, at constant massless velocity $(\forall)$, the momentum of a particle is zero only. It means that the particle does not need any external force to be at the massless velocity $(\forall)$ constantly. And the particle will be without any evolution for billion years if it is tuned to the massless velocity $(\forall)$ all the time. Thus, the human can be immortal.

\section{Can Any System Predict Future?}

Traditional concept of time is that, when a moment is in the future, it is probabilistic and when it becomes past, it is definite. But past, present and future are psychological divisions only. Because we cannot alter past, thus it is definite. And because we cannot anticipate future at present, it looks like probabilistic. But time has its own speed only; it does not depend on psychological divisions at all. For time, past, present and future are all same - just time only. Because of the serious lacking we have that we cannot anticipate the future which is fixed in any sense, we are unable to build a system which can anticipate future. Computers and artificial intelligence only resembles our brain only and nothing to do with anticipation of future. Because past, present and future are all the same time, uncertainty principle is invalid. 35 to $40 \%$ of our memory is unconscious mind, might be the reason, we cannot anticipate future. Unless we know, how to anticipate future, we cannot build a system that can anticipate future too. It might be a very simple task, but we have no idea how to do that. There are also psychological divisions on what we can do and what we cannot do. What we can do looks very simple to us and equally difficult what we cannot do. For time, nothing is difficult or easy, a task is a task only, not difficult task and not easy task either.

\section{Conclusion}

Only speed can help us to vary the rate of change of states of matter, in other words, evolution. Speed can alter evolution. Because we cannot build as massive particle like Earth which concerns with light years in space and 1000 years of time in one go, only option we are left with is the speed of a particle and acceleration to work with; both of them relates space with time. Speed is a very important property of matter that related space with time. This is the only function which has both independent variable space and time. Given all the information of speed along with space, time can be completely specified disregard of psychological divisions of time like past, present and future. Time is time only. Time is not at times, definite and the other times, probabilistic. Time is not that how we fix it. But time is that how we fit ourselves into it which is our psychological evaluation of time.

\section{Acknowledgment}

I am cordially grateful to Dr. Aparna Nath, Associate Professor and my PhD Guide, The department of Physics, National Institute of Technology, Agartala, India, for the epitome of inspiration and motivation to write this particular paper with perfection and accuracy. I am extremely thankful to her for all possible help she made to complete this paper. Also I am thankful to The Department of Physics of National Institute of Technology Agartala (NIT Agartala) for proper conduct and coordination.

\section{References}

[1] Debnath, Prasenjit, "Time Is Either Positive Evolution Or Negative Evolution Of Matter-Energy To Attain New State Of Equilibrium Or New State Of Stability: The Rate Of Change States Of Matter Is Evolution And Hence, Time." International Journal of Science and Research, Volume-6, Issue-6, Page -1785-1788.

[2] http://www.astronomy.ohio-state.edu/

[3] http://www.curious.astro.cornell.edu/ 


\section{International Journal of Science and Research (IJSR) \\ ISSN (Online): 2319-7064}

Index Copernicus Value (2015): 78.96 | Impact Factor (2015): 6.391

[4] Barrow, John D., Tipler, Frank J. "The Anthropic Cosmological Principle", Oxford University Press, ISBN 978-19-282147-8, LCCN 87028148, 1988.

[5] Cirkovic, M.M. "On The First Anthropic Argument in Astrobiology", Earth, Moon, and Planets. 91 (4):243254, doi:10.1023 /A:1026266630823, 2002.

[6] Cirkovic, M.M. "The Anthropic Principle and the Duration of Cosmological Past", Astronomical and Astrophysical Transactions. 23(6): 567-597, 2004.

[7] Roger Penrose, "Cycles of Time", Vintage Books, London, pp. 50-56.

[8] Stephen Hawking, "A Briefer History of Time", Bantam Books, London, pp. 1-49.

[9] Stephen Hawking, "Black holes and Baby Universes and other essays", Bantam Press, London 2013, ISBN 978-0-553-40663-4

[10] Stephen Hawking, "The Grand Design”, Bantam Books, London 2011

[11] Stephen Hawking, "A Brief History of Time", Bantam Books, London 2011, pp. 156-157. ISBN-978-0-55310953-5

[12] Stephen Hawking, "The Universe in a Nutshell", Bantam Press, London 2013, pp. 58-61, 63, 82-85, 90-94, 99, 196. ISBN 0-553-80202-X

[13] Stephen Hawking, "The Beginning of Time", A Lecture.

[14] Stephen Hawking, “Stephen Hawking's Universe: Strange Stuff Explained”, PBS site on imaginary time.

[15] Gerald D. Mahan, "Many-Particle Physics", Third Edition, Springer, 2000

[16] Uno Ingard, K "Fundamental of Waves \& oscillations", Cambridge University Press. P. 38, ISBN-0-521-33957XOxford: The British Academy, 1999

[17]A. Zee, "Quantum Field Theory in a Nutshell", Princeton University Press, 2003

[18] Storrs McCall, "A Model of the Universe", Oxford: Clarendon Press, 1994

[19] Craig Callender, "Time, Reality and Experience", Cambridge, UK: Cambridge University Press.

[20]Craig Callender, "Thermodynamic Asymmetry in Time", The Stanford Encyclopedia of Philosophy (Spring 2002 Edition)

[21] Storrs McCall, "A Model of the Universe", Oxford: Clarendon Press, 1994

[22] Robin Le Poidevin and Murray McBeath, "The Philosophy of Time" Oxford: Oxford University Press, 1993

[23] Whitrow, G., "The Natural Philosophy of Time". Oxford: Oxford University Press, 1961. (2nd edn., 1980.)

[24] Smart, J. J. C., "Problems of Space and Time". London: Macmillan, 1964

[25] Stephen Hawking, "A stubbornly persistent illusionThe essential scientific works of Albert Einstein", Running Press Book Publishers, Philadelphia, London 2011.

[26] William L.Craig, "Time and the Metaphysics of Relativity", Dordrecht: Kluwer Academic Publisher, 2001

\section{Author Profile}

Prasenjit Debnath, born in Agartala, Tripura, India on $15^{\text {th }}$ of March 1979. He is pursuing PhD degree in the Department of Physics in National Institute of Technology Agartala (NIT Agartala), India. 